\title{
Genome-wide dynamic network analysis reveals a critical transition state of flower development in Arabidopsis
}

\author{
Fuping Zhang ${ }^{1,2}$, Xiaoping Liü ${ }^{3}$, Aidi Zhang ${ }^{1}$, Zhonglin Jiang ${ }^{3}$, Luonan Chen ${ }^{3^{*}}$ and Xiujun Zhang ${ }^{1^{*}}$ (D)
}

\begin{abstract}
Background: The flowering transition which is controlled by a complex and intricate gene regulatory network plays an important role in the reproduction for offspring of plants. It is a challenge to identify the critical transition state as well as the genes that control the transition of flower development. With the emergence of massively parallel sequencing, a great number of time-course transcriptome data greatly facilitate the exploration of the developmental phase transition in plants. Although some network-based bioinformatics analyses attempted to identify the genes that control the phase transition, they generally overlooked the dynamics of regulation and resulted in unreliable results. In addition, the results of these methods cannot be self-explained.

Results: In this work, to reveal a critical transition state and identify the transition-specific genes of flower development, we implemented a genome-wide dynamic network analysis on temporal gene expression data in Arabidopsis by dynamic network biomarker (DNB) method. In the analysis, DNB model which can exploit collective fluctuations and correlations of different metabolites at a network level was used to detect the imminent critical transition state or the tipping point. The genes that control the phase transition can be identified by the difference of weighted correlations between the genes interested and the other genes in the global network. To construct the gene regulatory network controlling the flowering transition, we applied NARROMI algorithm which can reduce the noisy, redundant and indirect regulations on the expression data of the transition-specific genes. In the results, the critical transition state detected during the formation of flowers corresponded to the development of flowering on the 7th to 9th day in Arabidopsis. Among of 233 genes identified to be highly fluctuated at the transition state, a high percentage of genes with maximum expression in pollen was detected, and 24 genes were validated to participate in stress reaction process, as well as other floral-related pathways. Composed of three major subnetworks, a gene regulatory network with 150 nodes and 225 edges was found to be highly correlated with flowering transition. The gene ontology (GO) annotation of pathway enrichment analysis revealed that the identified genes are enriched in the catalytic activity, metabolic process and cellular process.
\end{abstract}

Conclusions: This study provides a novel insight to identify the real causality of the phase transition with genomewide dynamic network analysis.

Keywords: Time-course gene expression data, Gene regulatory network (GRN), Dynamical network biomarker (DNB) , Phase transition, Flower development

\footnotetext{
*Correspondence: Inchen@sibs.ac.cn; zhangxj@wbgcas.cn

${ }^{3}$ Key Laboratory of Systems Biology, Innovation Center for Cell Signaling Network, Institute of Biochemistry and Cell Biology, Shanghai Institutes for Biological Sciences, Chinese Academy of Sciences, Shanghai 200031, China ${ }^{1}$ Key Laboratory of Plant Germplasm Enhancement and Specially Agriculture, Wuhan Botanical Garden, Chinese Academy of Sciences, Wuhan 430074,

China

Full list of author information is available at the end of the article
}

(c) The Author(s). 2019 Open Access This article is distributed under the terms of the Creative Commons Attribution 4.0 International License (http://creativecommons.org/licenses/by/4.0/), which permits unrestricted use, distribution, and reproduction in any medium, provided you give appropriate credit to the original author(s) and the source, provide a link to the Creative Commons license, and indicate if changes were made. The Creative Commons Public Domain Dedication waiver (http://creativecommons.org/publicdomain/zero/1.0/) applies to the data made available in this article, unless otherwise stated. 


\section{Background}

The flowering plants undergo a succession of developmental phases during their life cycle including germination, seedling, flowering and fruiting. As a special pattern of plant development, phase transition is crucial for the survival and reproduction of the plant, and the failure implementation of phase transition will result in the dysfunction of development [1-3]. The phase transitions related to plant development include the seed-to-seedling transition [4], the juvenile-to-adult vegetative transition [5], the vegetative-to-reproductive transition [6], the heterotrophic-to-autotrophic transition [7], the initiation-tomaturation floral transition [8], and so on. These developmental phase transitions form the main functional mechanisms in plants [9].

Among the developmental phase transitions, flowering transition has been extensively studied by the experimental biologists because flowering is an irreversible transformation from vegetative to reproductive growth, an important qualitative process, a key stage of plant develop ment, and a reproduction for offspring [10]. The flowering process include three phases, i.e. the floral induction phase, the floral primordia phase, and the floral organs development phase [8]. During these phases, a series of genes such as flowering time controlling genes, meristem identity genes and floral organ identity genes are involved in the regulation of flowering transition [11].

To understand the mechanism of flowering transition, a series of intensive studies on flower development were implemented in recent years and the results provided some crucial clues [12-15]. For example, it is reported that flower development is firstly characterized by dramatic changes in morphology such as floral patterning, floral organ size and floral organ specification. These changes are regulated by a great number of regulators like transcriptional factors and miRNAs $[8,16]$. The growth cone is transformed from differentiation of leaves to differentiation of flower buds in the ontogeny of higher plant, which marks the transition from vegetative growth to reproductive growth or the beginning of flower development [11].

In recent years, it has become increasingly clear that the phase transitions were controlled by distinct genetic circuit incorporating endogenous and environmental cues, such as the interaction between NF-YC and CLF, the interaction between miR156 and miR173 [9], and so on. These genes are involved the regulation of phase transition by undergoing regular changes to form a complex gene regulatory network (GRN) [11]. The flowering mechanism of the model plant Arabidopsis is relatively clear with a flowering gene regulatory network involving signal transduction networks such as photoperiod, autonomous, vernalization, and gibberellin pathways [17]. More and more researches demonstrate that the flowering developmental gene regulatory networks provide an important breakthrough for better understanding the inherent mechanism of flowering transition.

Attribute to the advances of next-generational sequencing technology, bioinformatics and computational biology expedited the process of biological research by ge nome-wide transcriptome analysis [18]. Transcriptome analysis provides a perfect approach to study biological problem because it gives not only a global view of gene expression patterns including biological function enrichment but also a predictive dimension by identifying a set of co-expressed genes $[19,20]$. With above advantages, bioinformatics analysis of high-throughput transcriptome data provides a powerful tool to address the issues in exploring the mechanism of the phase transition in a genome-wide scale [21].

The transcriptome analysis by predicting GRN has been used to discover the regulatory mechanism in plants [22]. Different types of tools have been developed to infer large-scale GRNs from the gene expression data, such as correlation-based methods [23], mutual informationbased method [24, 25] and regression-based methods [26-28]. Our group previously have developed some GRN inference tools such as NARROMI [29], PCA-CMI [30] and CMI2NI [31, 32]. These methods have greatly improved the accuracy of GRN inference by reducing the noisy, redundant and indirect regulations.

Recently, the predictive GRN was used to analyze the phase transition and some novel genes controlling the seed-to-seedling phase transition in Arabidopsis were identified [19, 33]. Not only that, the Arabidopsis floral transition process was deciphered by integrating a protein-protein interaction network and gene expression data [34]. For the traditional analysis methods, it is difficult to catch up the truth of complex phase transitions with the traits of temporal and spatial dynamics with polygene interactions. Moreover, the research has focused on the mechanism of flower development under undifferentiated stem cells but not the mechanism of floral organ transformation to maturation [35]. In addition, the current methods have not fully used the dynamics character of phase transition and not necessarily catch the real regulators controlling the phase transitions [36]. While the time-course transcriptome data with multiple replicates provide the materials for the bioinformatics analysis to identify the regulators of phase transition [37, 38].

With more and more time-course high-throughput transcriptome data available, some dynamic network analysis-based bioinformatics tools have been developed and widely used to study the complex biological mechanism [39, 40]. For example, the dynamic network biomarker identification tool, named DNB, was developed to detect the biomarker [41]. In this method, a 
complex biological process (e.g., differentiation processes, aging processes, and phase transition of the cell cycle, etc.) is divided into three phases or states, i.e. the before-transition state, the critical transition state, and the after-transition state [42]. In a focal system, a drastic or qualitative transition from the critical transition state to the post-transition state corresponds to a bifurcation point in the dynamical systems theory [43]. When the system is near to a critical point, a dominant group of biomarkers become bifurcate. The biomarkers can be defined by three conditions: isolated subnetwork or functional module, high fluctuation of the members, strong correlation between any pair of its members while weak correlation between members and nonmembers [44]. Dynamic network analysis with a solid theory support has been successfully applied to real biological data [43-45].

In this work, to provide a new insight into the flowering developmental transition, we performed a genome-wide dynamic network analysis on the time-course gene expression data in Arabidopsis. The dataset with 14 stages of flowering from initiation to maturity and 3 replicates for each stage provides a dynamic process of flowering development. The genes that control the phase transition can be identified by the difference of weighted correlations between the genes interested and the other genes in the global network. To construct the gene regulatory network controlling the flowering transition, we applied NARROMI algorithm which can reduce the noisy, redundant and indirect regulations on the expression data of the transition-specific genes. The results were validated by biological experimental analysis and the predicted transition state is consistent with the real transition state of the flowering in phenotype. We also performed a similar analysis of temporal gene expression profiling dataset of rice flower development to support the conclusions drawn. Ultimately, we detected a critical transition state of flower development in rice. This work provides a novel insight into the identification of the transition state and the key causal genes that control flowering transition by dynamic network analysis of time-course gene expression data.

\section{Results}

A dynamic network biomarker(DNB) model for detecting critical transition state of complex biological systems

In the phase transition model, a complex biological process (e.g., differentiation processes, aging processes, and changes in the phases of the cell cycle) can be generally divided into the three stages, i.e. the before-transition state, the critical transition state, and the after-transition state [42]. The before-transition state is relatively stable but may change gradually because of certain internal and external motivators (Fig. 1). The critical transition state can be understood as the limit of the before-transition state just before a critical transition. Not only that, but it can be easily reverted to the before-transition state by the appropriate external interventions and thus it is considered reversible. While the after-transition state is stable and irreversible even with intensive interventions. Throu gh the identification of dynamic network biomarkers (DNBs), we can control the complex system to avoid the development of the system toward the bad state. In other words, controlling the "causes" of the development of the system can avoid the occurrence of "effects" or change the shape of "effects". It is crucial to crush the source of trouble in the egg to achieve the ideal effect of achieving effective control of complex systems at a lower cost.

The theoretical basis for detecting DNBs was summarized by the following conditions (Fig. $1 \mathrm{~b}$ and c). Briefly, DNB is an observable subnetwork composed of a set of special molecules in the original system which satisfies the following four requirements only at the critical state [46]:

1. DNB molecules or markers are highly fluctuating, i.e. deviations of the DNB members(genes) increase drastically (high $\mathrm{SD}_{d}$ ).

2. All members of DNBs are highly co-expressed, i.e. correlations among DNB members become stronger (high $\mathrm{PCC}_{d}$ ).

3. DNB members are almost independent of non-DNB members. DNB is an isolated subnetwork or functional module, i.e. the correlation between any DNB molecules and other non-DNB decreases (low $\mathrm{PCC}_{o}$ ).

4. There are no drastic deviations or correlations among all non-DNB molecules in the system.

To detect a reliable and unambiguous signal of the critical transition state, a composite index(CI) is proposed as follows

$$
\mathrm{CI}=\left(\mathrm{SD}_{d} \cdot \mathrm{PCC}_{d}\right) / \mathrm{PCC}_{o},
$$

where $\mathrm{SD}_{d}$ and $\mathrm{PCC}_{d}$ are average standard deviation(SD) and average Pearson correlation coefficient(PCC) of all molecules in DNB module $d$ respectively, while $\mathrm{PCC}_{o}$ is the average correlation between molecules in $d$ and others that are not in $d$. When a biological system approaches a critical transition state, CI provides a reliable and significant early-warning signal. Among all the responsive $\mathrm{CI}$ modules, the maximum one is most likely to be the DNB that corresponds to the critical stage of the system.

\section{Pipeline of genome-wide dynamic network analysis}

To provide a new insight into the flowering developmental transition, we performed a genome-wide dynamic network analysis on the time-course gene expression data in 


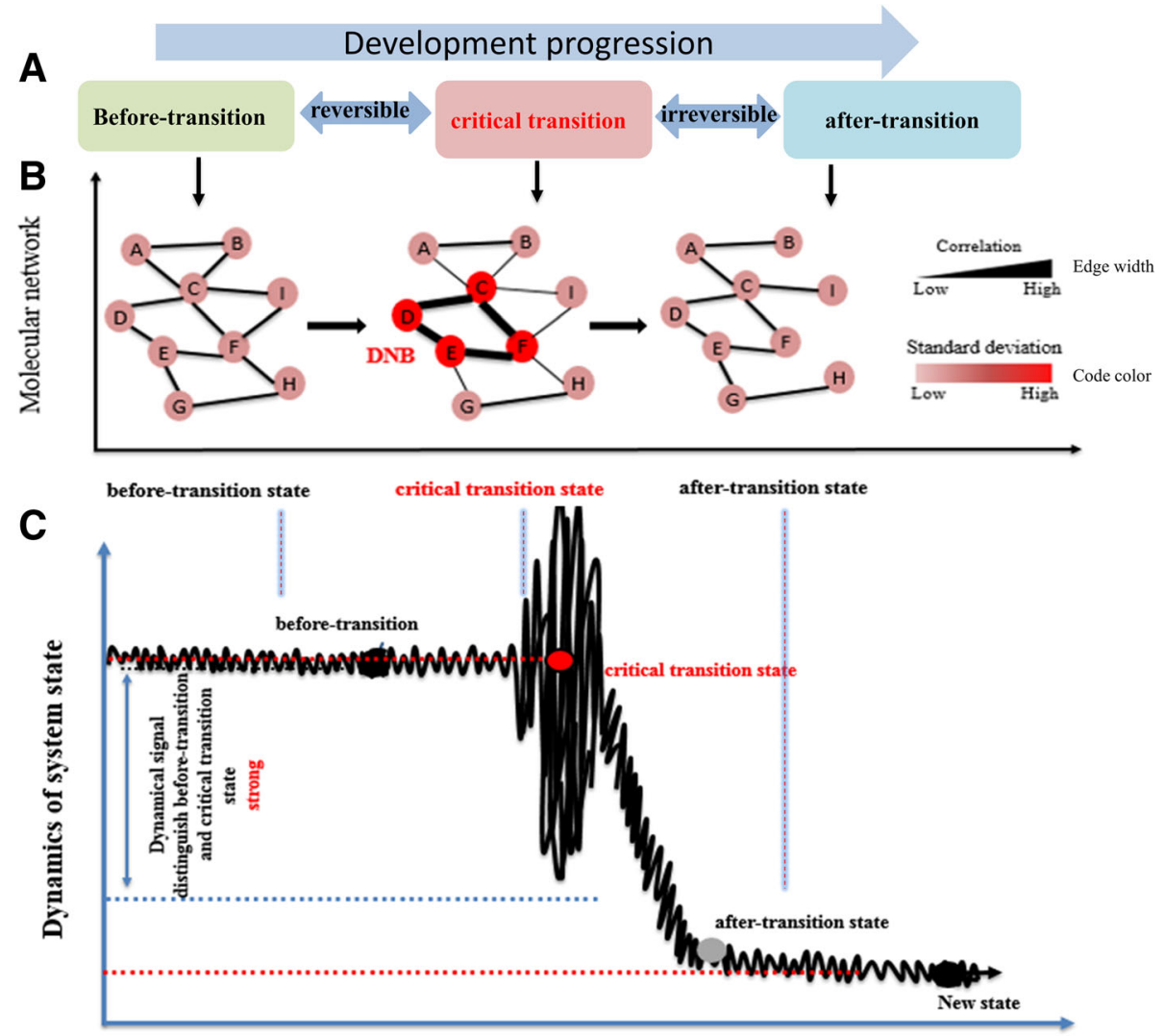

Fig. 1 The outline representation of critical transition state by DNB based on time-series gene expression data. a The progression of Arabidopsis flower development from the time of initiation to maturation can be divided into three states, i.e., the before-transition state, the critical transition state, and the after-transition state. A system at the before-transition state or the after-transition state is stable with high resilience. $\mathbf{b}$ In the critical transition state, deviations of the DNB members increase drastically, and the correlation between any two molecules increases. This critical phenomenon does not appear at the before-transition and the after-transition states. $\mathbf{c}$ The DNB members show large fluctuations in their expressions at the critical transition state, compared with smaller fluctuations of the expressions at the before- or after- transition states

Arabidopsis. The dataset with 14 stages of flowering from initiation to maturity and 3 replicates for each stage provides a dynamic process of flowering development. However, the dynamic network analysis to effectively reveal the critical developmental stage with a key sub-network is still a challenge [8]. In addition, the data with matched case and control samples are not available. Therefore, the effective data processing become crucial to identify the DNBs for the flower developmental transition.

Previous studies have shown that Arabidopsis flowers are sequentially activated so that the flowers in their inflorescence are at different stages of development [47]. It also has been found that the top-head flowers of the Arabidopsis inflorescence remain quite synchronized throughout the flower development and therefore the time-series gene expression data of flowering could be obtained [8]. To overcome the lack of data with the matched case and control samples, the sample in former time point was designated as the control sample, and the neighbor later sample was designed as case sample. And then the 14 different developmental stages from initial to mature were divided into 13 case-control combinations with which we can detect the phase transition of Arabidopsis flower development (Fig. 2a).

The DNB model which is powerful than the traditional differential expression analysis was applied to detect the critical differentiation state of flower development from the time of initiation to maturation in Arabidopsis (Fig. 2b). NARROMI algorithm which can reduce the noisy, redundant and indirect regulations on the expression data of the transition-specific genes was used to construct the gene regulatory network controlling the flowering transition (Fig. 2c) [29]. With the identified DNBs, key regulatory factors and metabolic pathways closely related to the phase transition of Arabidopsis flower development from the time of initiation to maturation were analyzed (Fig. 2d).

The identified critical transition state in Arabidopsis flower development

Identifying the critical differentiation stage or critical transition state of Arabidopsis flower development from 




Fig. 2 The flow diagram for revealing the critical transition state. a With samples in previous and the current time points designated as the control and case sample respectively. The 14 different developmental stages from initial to mature were divided into 13 combinations. b We applied DNB method to reveal a critical differentiation state of Arabidopsis flower development by comparing the control and case samples. c To construct the gene regulatory network controlling the flowering transition, we applied NARROMI algorithm on the expression data of the transition-specific genes. The NARROMI algorithm removes noisy regulations with low pair-wise correlations and redundant regulations from indirect regulators using ordinary differential equation-based recursive optimization (RO) and information theory-based mutual information (MI), respectively. The dotted line without arrowhead denotes non-regulation (redundant), the dotted arrow denotes the indirect regulation (redundant) and the solid arrow denotes the true regulation. $\mathbf{d}$ We also analyzed key regulatory factors and key metabolic pathways which were closely related to the phase transition of Arabidopsis flowering development from the time of initiation to maturation time

the time of initiation to maturation is crucial to clarify the molecular mechanism that regulates plant flowering. However, the traditional methods based on differential expression analysis failed to detect the critical state due to the lack of significant differential expressions of molecules in the before-transition and critical transition states. To overcome this problem, the DNB model was developed to measure collective fluctuations of molecules taking place of the traditional differential expression analysis (see Methods). Traditional methods often rely on the differential expressions of molecules, while the DNB model uses both differential 
correlations and differential deviations among molecules $[41,48]$. Despite of the weak differential expression among genes in the before-transition state and the critical transition state, significant differential correlations and deviations among genes existed in these two states.

We implemented the DNB model on the Arabidopsis flower development datasets (NCBI access no. GSE64581) to identify the critical transition and the genes of flower development from the time of initiation to maturation. Specifically, we compared the samples in previous time point as the control sample with the samples in current time point as the case sample. Ultimately, the 14 different developmental stages from initial to mature were divide into 13 sampling time points. The critical transition state of Arabidopsis flowering was detected in the before-transition and after-transition state at the 11th sampling point of the flower formation shown by the red star (Fig. 3).

The 11th sampling time point shown in Fig. 3 corresponded to the comparison of growth on the 7th to 9th day of Arabidopsis flower development. Moreover, the development of flower inflorescence was largely synchronous until day 7. For the later time-points, only the development of flowers at the tip of the inflorescence (arrowheads) remained synchronized after phenotypic assessment (Fig. 3) [8]. In order to verify the biological and statistical significance of the identified DNBs, we conducted bootstrap analysis and the results showed that the identified DNBs for flowering development in Arabidopsis were highly significant compared with the randomly chosen gene sets (Additional file 1: Figure S4).

\section{The identified genes controlling the transition of Arabidopsis flower development}

Ryan et al. revealed that the development of Arabidopsis flower inflorescence was largely synchronous until day 7 . After that, only the development of flowers at the tip of the inflorescence remained synchronized after phenotypic assessment [8]. As result of our analysis, the predicted critical transition state from timepoint 7 th to 9th day was consistent with their experimental results. In other words, the critical transition state detected during the formation of flowers corresponded to the development of flowering in Arabidopsis. All identified DNB members corresponding to the critical state of flower formation were listed in Additional file 2: Figure S1, and the detailed description of DNB members was listed in Additional file 3: Figure S2. Functional categories for up- and down-regulated genes in DNB module were shown in Table 1 , these genes were crucial for flowering transition. Moreover, functional categories for other genes in DNB module were shown in Additional file 4: Table S1.

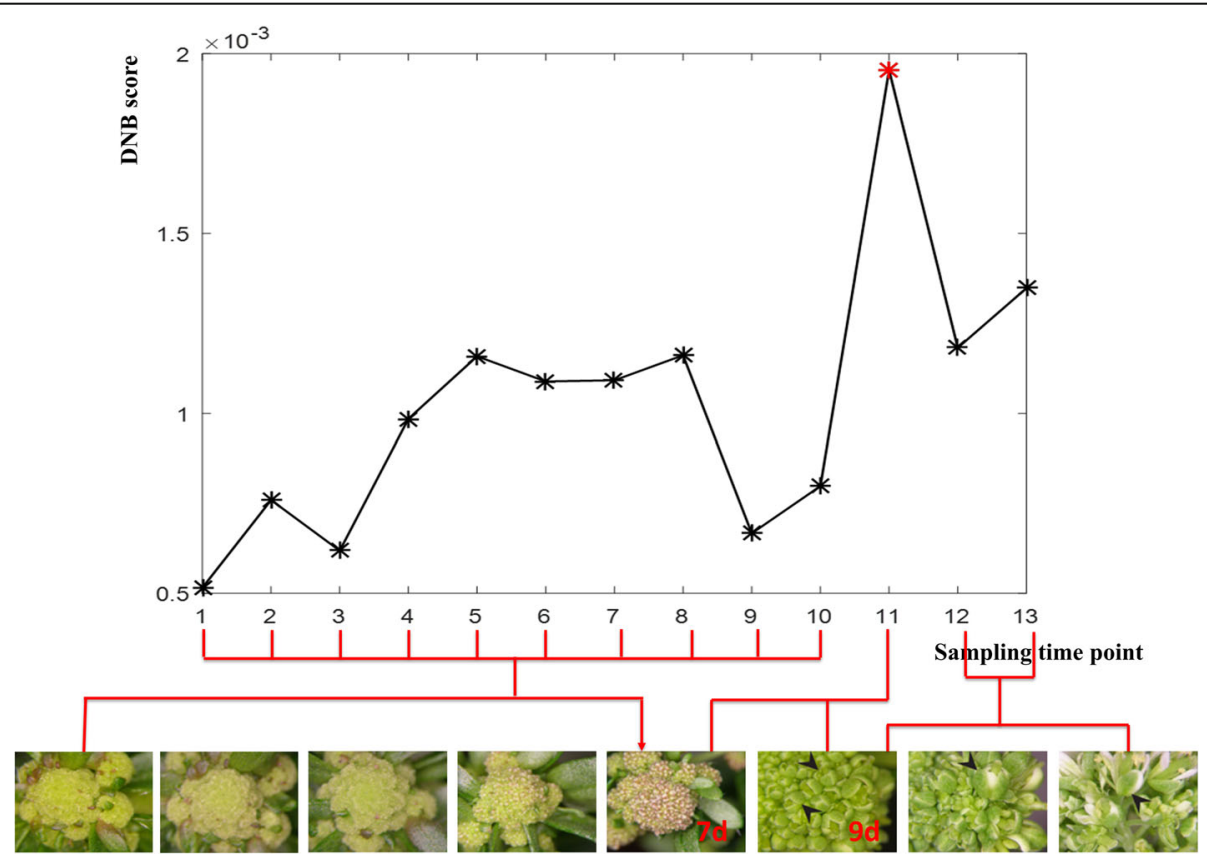

Fig. 3 Identification of the critical transition state of flower development in Arabidopsis thaliana. The DNB scores at 13 sampling time points is shown in the FIGURE. For the black curve, the DNB score increased sharply from the 10th point and reached the peak at the 11th point. The 11th sampling time point annotated by the red star is designated as a critical transition state, which corresponds to the comparison of growth on the 7th to 9th day of Arabidopsis flower development 
Table 1 Functional categories for up- and down-regulated genes in the DNB module of Arabidopsis flower development

\begin{tabular}{|c|c|c|c|}
\hline Gene name & Fold change & Transition state & Description \\
\hline \multicolumn{4}{|c|}{ Transcription factors } \\
\hline RAP2.6 & 0.73 & down-regulated & AP2/B3-like, related to AP2 6(RAP2.6) \\
\hline AGL46 & 0.19 & down-regulated & MADS box transcription factor \\
\hline AT2G31370 & 1.74 & up-regulated & Basic-leucine zipper transcription factor \\
\hline AT5G46030 & 2.12 & up-regulated & transcription elongation factor-like protein \\
\hline AT4G33280 & 0.71 & down-regulated & AP2/B3-like transcriptional factor family protein \\
\hline AT1G21580 & 1.66 & up-regulated & Zinc finger $\mathrm{C}-\times 8-\mathrm{C}-\times 5-\mathrm{C}-\times 3-\mathrm{H}$ type family \\
\hline ZAP1 & 4.05 & up-regulated & zinc-dependent activator protein-1 \\
\hline WRKY36 & 0.07 & down-regulated & WRKY DNA-binding protein \\
\hline AT1G59675 & 1.21 & up-regulated & F-box family protein \\
\hline NAP & 2.37 & up-regulated & NAC-like, activated by AP3/PI(NAP) \\
\hline AT1G76590 & 1.63 & up-regulated & PLATZ transcription factor family protein \\
\hline RIE1 & 0.28 & down-regulated & RING-finger protein for embryogenesis \\
\hline AT4G25610 & 0.35 & down-regulated & $\mathrm{C} 2 \mathrm{H} 2$-like zinc finger protein \\
\hline \multicolumn{4}{|c|}{ Protein kinase activity } \\
\hline AT3G46270 & 3.83 & up-regulated & receptor protein kinase-like protein \\
\hline \multirow[t]{2}{*}{ AT3G15240 } & 3.61 & up-regulated & Serine/threonine-protein kinase WNK \\
\hline & & & (With No Lysine)-like protein \\
\hline AT1G67720 & 0.42 & down-regulated & Leucine-rich repeat protein kinase family protein \\
\hline AT3G23310 & 0.75 & down-regulated & AGC kinase family protein \\
\hline \multicolumn{4}{|c|}{ Ras signaling pathway } \\
\hline AGL46 & 0.19 & down-regulated & MADS box transcription factor \\
\hline \multicolumn{4}{|c|}{ Plant hormone signal transduction } \\
\hline $\mathrm{SHY} 2$ & 0.61 & down-regulated & AUX/IAA transcriptional regulator family protein \\
\hline AIR12 & 0.79 & down-regulated & auxin-induced in root cultures-like protein \\
\hline IAA18 & 1.98 & up-regulated & indole-3-acetic acid inducible 18 \\
\hline \multicolumn{4}{|c|}{ ABA signaling pathway } \\
\hline HIS1-3 & 0.79 & down-regulated & histone $\mathrm{H} 1-3$ \\
\hline $\mathrm{SLAH2}$ & 1.22 & up-regulated & SLAC1 homologue 2 \\
\hline AT4G25610 & 0.35 & down-regulated & $\mathrm{C} 2 \mathrm{H}$ 2-like zinc finger protein \\
\hline AT4G33280 & 0.71 & down-regulated & AP2/B3-like transcriptional factor family protein \\
\hline AT1G59675 & 1.21 & up-regulated & F-box family protein \\
\hline
\end{tabular}

\section{A more reliable gene co-expression network controlling critical transition}

A DNB module containing 233 genes about Arabidopsis flower development from the time of initiation to maturation was detected using the DNB model [41]. With the gene expression data of these transition-specific genes, we used NARROMI algorithm which can remove the redundant and indirect regulations to construct the gene co-expression network controlling the flowering tran sition. Network inference file was listed in Additional file 5: Table S3, and we got two new files by processing the file. The degree of correlation between each pair of DNB members of Arabidopsis flower development was listed in Additional file 6: Table S4, and the node properties file for the network was listed in Additional file 7: Table S5.

By applying the NARROMI approach to the 233 genes of the DNB module, we constructed a more accurate and reliable gene regulatory network of the Arabidopsis flowering transition. The file of gene regulatory network was listed in Additional file 8: Table S6. Ultimately, the members of DNB module and the correlations among members were visualized in a molecular network consisting of 150 nodes with 225 edges. In the network, node color reflects the standard deviation of the corresponding genes and the strength of correlations reflected 




Fig. 4 DNB members and correlations among these molecules were visualized in molecular networks by using Cytoscape. Node color reflects the standard deviation of the corresponding genes. The strength of correlations is represented by edge width, where a wider edge corresponds to a higher correlation. For clarity, the strength of correlations was also reflected by edge color. a A more reliable co-expression gene regulatory network of the Arabidopsis floral transition. b Three key subnetworks in DNB given separately

by edge width, where a wider edge corresponds to a higher correlation (Fig. 4a). Three key subnetworks in the DNB module are clustered separately because the standard deviation of the corresponding genes and the strength of correlations in these subnetworks were significantly different, and we deemed these distinct regions as the most representative of this critical transition (Fig. 4b).

\section{Functional classification of DNB members during the critical transition state}

The critical transition state during the formation of flowers was identified by DNB approach, and it corresponded to the development of flowering on the 7 th to 9th day in Arabidopsis. To evaluate the potential functions of DNB members, GO assignments were used to classify the functions of DNB members during the critical transition of Arabidopsis flower development.

In the biological process category, two GO terms, i.e. small molecule catabolic process and plant-type cell wall biogenesis were enriched significantly in most DNB members (Fig. 5a). In the cellular component category, there were plenty of DNB members associated with AP-type membrane coat adaptor complex, secondary cell wall, and intrinsic component of mitochondrial inner membrane (Fig. 5b). In the molecular function category, ion antiporter activity was enriched significantly in most genes of the DNB module, and phosphate ion transmembrane transporter activity, serine-type endopeptidase inhibitor activity and L-ascorbic acid binding were similarly enriched in the DNB module (Fig. 5c). 


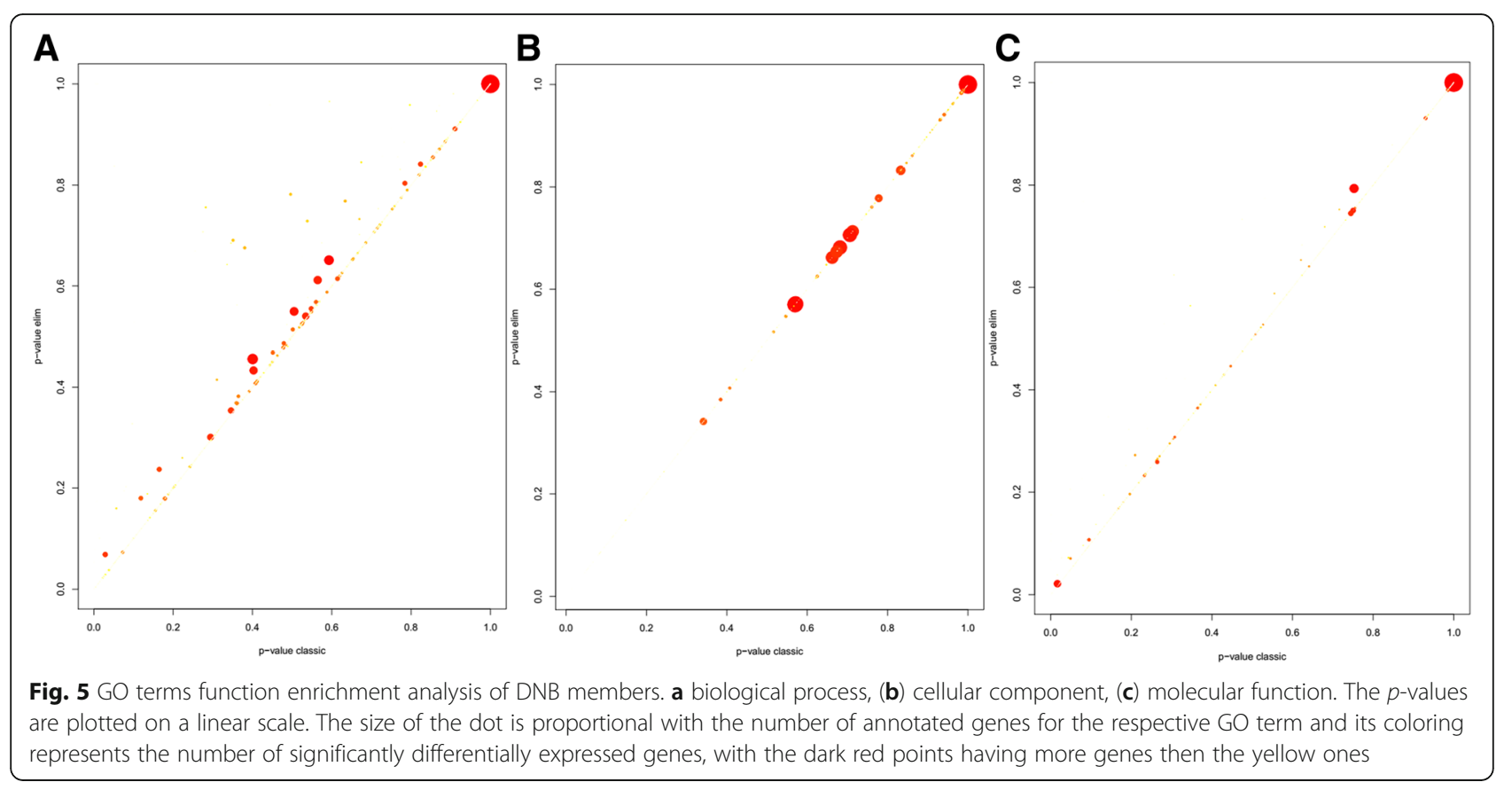

All the other overrepresented GO terms of DNB members in the three GO categories (biological process, cellular component, and molecular function) were listed in Table 2. And the metabolic pathways were listed in Additional file 9: Table S2. To further analyze key regulatory factors that control the phase transition from initiation to maturation in Arabidopsis flower development, we inferred a transcriptional module that links key regulatory factors with their potential targets (Fig. 6).

\section{The detected key genes of the critical transition}

We not only discovered a critical transition which occurred between the seventh and ninth days of Arabidopsis flower development, but also found that 24 genes in this DNB module participate in stress reaction process, as well as in other floral-related pathways (Fig. 6b). Biological and abiotic stresses negatively affect plant growth and development including flowering and thus reduce productivity [49]. Adversity related genes included disease-, ABA-, JA-, drought-, low temperature and salt-rela ted genes, i.e. CBP20, NAP, AT4G33280, RAP2.6, ECA1, AT5G38760, WRKY36, HIS1-3, AT1G73490, RIE1, AT1 G52650, AT4G08520, AT1G59675, etc. Many of these genes such as RIE1, ECA1 and NAP were predominantly expressed from or after the 9th day which is the pollen formation stage. Thus, these genes might be play a vital role in the formation of microspores and the differentiation of pollen grains to a large extent.

The cap-binding protein complex $(\mathrm{CBC})$ plays an important role in RNA metabolism because the CBC binds to the caps of all RNA polymerase II transcripts. As one subunit of the Arabidopsis thaliana CBC, the Cap Binding
Protein 20 (CBP20) was determined to be involved in normal plant growth and development as well as RNA metabolism [50]. Studies have found that Arabidopsis cbp20 null mutant exhibited abnormal development of leaves and flowers and showed increased sensitivity to salt stress, which suggests that CBP20 has a synergistic effect in salt stress response [51]. Moreover, the drought-tolerant cbp20 mutant could maintain normal growth and development under drought stress, which might also point to a new cellular output mechanism as targets of the ABA regulatory pathway [52]. The plant specific NAM/ATAF1/ 2/CUC2 (NAC) transcription factors play important roles in abiotic stress-response signaling. We found that two NAC-like genes named NAP and NAP57 (activated by AP3/PI) as two of the important DNB members involved in a trifurcate feed-forward pathway of the drought stress response and their expression at the critical transition state was different from other periods.

Moreover, we also found NAP12 to be involved in the gametogenesis of Arabidopsis with two genes of ECA1 (Early Culture Abundant 1) gametogenesis-related family genes, AT5G60945 and AT5G36657. The ECA1 family proteins can be activated transcriptionally during the transition of microspores from the gametophytic to the embryogenic pathway. In addition, RIE1 and LEA (AT5G38760), two genes in the DNB module were found to be involved in the embryonic development of Arabidopsis. A RIE1 gene encoding a RING-H2 zinc-finger protein was identified in Arabidopsis, and it might be a membrane-associated protein, possibly relating to chloroplast. The late embryogenesis Abundant (LEA) protein family plays a role in drought 
Table 2 Significance of GO terms obtained by R package topGO

\begin{tabular}{|c|c|c|c|c|}
\hline $\mathrm{GO}$ & GO.ID & Term & Annotated & $P$-value \\
\hline \multirow[t]{10}{*}{ biological process } & GO:0044282 & small molecule catabolic process & 196 & 0.0044 \\
\hline & GO:0009832 & plant-type cell wall biogenesis & 174 & 0.00428 \\
\hline & GO:0006643 & membrane lipid metabolic process & 101 & 0.00801 \\
\hline & GO:0009640 & photomorphogenesis & 95 & 0.00081 \\
\hline & GO:0032101 & regulation of response to external stimu... & 85 & 0.00713 \\
\hline & GO:0090691 & formation of plant organ boundary & 16 & 0.00943 \\
\hline & GO:0010244 & response to low fluence blue light stimu... & 9 & 0.00719 \\
\hline & GO:0010264 & myo-inositol hexakisphosphate biosynthet... & 8 & 0.00617 \\
\hline & GO:0000967 & rRNA 5'-end processing & 7 & 0.00401 \\
\hline & GO:0052746 & inositol phosphorylation & 5 & 0.00133 \\
\hline \multirow[t]{10}{*}{ cellular component } & GO:0030119 & AP-type membrane coat adaptor complex & 18 & 0.015 \\
\hline & GO:0031304 & intrinsic component of mitochondrial inn... & 18 & 0.052 \\
\hline & GO:0009531 & secondary cell wall & 16 & 0.053 \\
\hline & GO:0005880 & nuclear microtubule & 7 & 0.031 \\
\hline & GO:0016442 & RISC complex & 5 & 0.047 \\
\hline & GO:0031332 & RNAi effector complex & 5 & 0.047 \\
\hline & GO:0034098 & VCP-NPL4-UFD1 AAA ATPase complex & 3 & 0.014 \\
\hline & GO:0032541 & cortical endoplasmic reticulum & 2 & 0.022 \\
\hline & GO:0000312 & plastid small ribosomal subunit & 2 & 0.041 \\
\hline & GO:0005971 & ribonucleoside-diphosphate reductase com... & 2 & 0.051 \\
\hline \multirow[t]{10}{*}{ molecular function } & GO:0099516 & ion antiporter activity & 94 & 0.0125 \\
\hline & GO:0015114 & phosphate ion transmembrane transporter ... & 26 & 0.0019 \\
\hline & GO:0004867 & serine-type endopeptidase inhibitor acti... & 22 & 0.0018 \\
\hline & GO:0031418 & L-ascorbic acid binding & 13 & 0.008 \\
\hline & GO:0048038 & quinone binding & 10 & 0.0031 \\
\hline & GO:0005242 & inward rectifier potassium channel activ... & 10 & 0.013 \\
\hline & GO:0016639 & oxidoreductase activity, acting on the C... & 6 & 0.0041 \\
\hline & GO:0050284 & sinapate 1-glucosyltransferase activity & 4 & 0.004 \\
\hline & GO:0004148 & dihydrolipoyl dehydrogenase activity & 4 & 0.0069 \\
\hline & GO:0016629 & 12-oxophytodienoate reductase activity & 3 & 0.0082 \\
\hline
\end{tabular}

stress tolerance. How to improve reproductive development by this transition depended on the interactions between regulatory factors. These regulators were important for signal transduction to control the transition of Arabidopsis flower development.

The APETALA2 (AP2) transcription factors (TFs) plays an important dynamic role in the embryo development, seedling built, flowering as well as stress response process $[53,54]$. Two genes including RAP2.6 and AP2/B3-like (AT4G33280) were identified by DNB method. The Arabidopsis transcription factors RAP2.6 and AP2/ERF were found to be involved not only in ABA, salt and drought stress responses, but also in stamen emergence [55]. WRKY genes are a family of regulatory genes isolated from plants. WRKY proteins encoded by WRKY genes constitute a large family of plant-specific transcription factors. WRKY36, one of the WRKY gene family members, was identified in DNB module. Recent research found that UVR8 interacts with WRKY36 to regulate HY5 transcription and hypocotyl elongation in Arabidopsis, while we found that WRKY36 plays an important role in the critical transition [56].

As result of our analysis, 233 genes were identified to be highly fluctuated at the transition state and formed the DNB module. More importantly, a high percentage of genes with maximum expression in pollen was detected, which were predominantly expressed from or after the 9th day. For example, we found that two NAC-like genes named NAP and NAP57 (activated by AP3/PI) as two of the important DNB members were involved in a trifurcate feed-forward pathway of the drought stress response and they were up-regulated at the critical transition state [57]. 


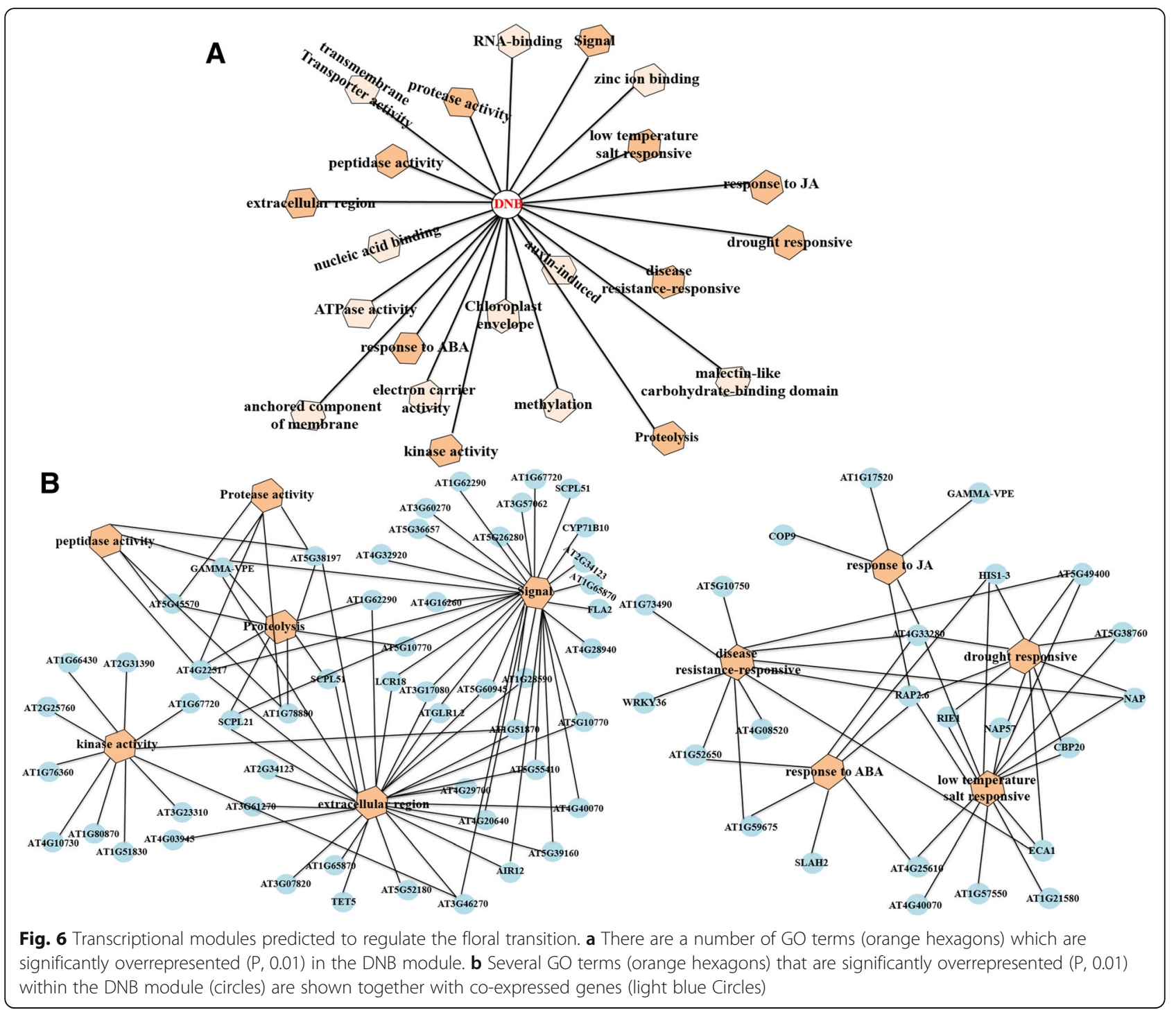

Numerous studies have shown that class B MADS-box genes (AP3/PI) are crucial for stamen development [58]. Thus, the critical transition state detected during the formation of flowers was regarded as the pollen formation stage.

Some genes involved in cell differentiation were detected in this DNB module with predominant expression at late stages of flower development. These genes were predominantly expressed from or after the 9th day which is the pollen formation stage. For example, the regulator of ovule and seed development SEEDSTICK (STK) was significantly upregulated between days 7 and 9 [49]. DUO POLLEN1 (DUO1), a regulator of male germline development, was found for the first time to be expressed at the same stage [50]. And thus, these genes may be involved to a large extent in the differentiation of microspores into pollen grains. Gene Ontology (GO) analysis also showed that secondary cell wall synthesis related genes were enriched significantly in this critical transition (GO:000 9531). The tapetum was developed from the secondary cell wall, which was the important component of pollen [59]. While many changes in gene expression may be owing to the activation of specific gene sets during the late pollen development, genes with significant regulatory functions such as genes coding APETALA2 (AP2) transcription factors and MADS-domain proteins often show intermittent expression at various stages of flower development [54, 55].

Genes involved in different plant hormone responses such as abscisic acid, auxin, and jasmonic acid were also detected as enriched significantly in this DNB module. This discovery is consistent with the known roles of these hormones in various biological processes in the late-stage flower development, such as the formation of stamen and 
pollen as well as the maturation of petals [60]. For example, AGL46 (AT2G28700), encoding the Arabidopsis MADS-box transcription factor was found to be involved in the CCKR signaling map, gonadotropin-releasing hormone receptor pathway, PDGF signaling pathway, interleukin signaling pathway, Ras Pathway and p38 MAPK pathway by KEGG analysis. Studies have shown that members of the MADS-box gene family play vital roles in flower development from early determination of floral meristem identity to later determination of floral organ primordial identity [58]. In a nutshell, the critical transition state detected during the formation of flowers and key regulators identified in the transition might answer the complex mechanisms of floral organ formation.

\section{A critical transition state of flower development in Rice}

To support the conclusions drawn, a similar analysis was performed on the flowering development data in rice. The dataset with spatio-temporal gene expression profiling throughout entire growth in rice was downloaded from the NCBI Gene Expression Omnibus (GEO) database under the accession number GSE21396 (www.ncbi.nlm.nih.gov/geo). The dataset include 15 time-points and 3 replicates for flowering development from initiation to maturity in rice [61]. To overcome the lack of distinction between the case sample and the control sample in this dataset, we compared the samples in both the previous and current time points. The samples in previous and current time points were designated as the control and case samples respectively. Thus, 15 different developmental stages from initial to mature were divided into 14 combinations for the detection of the critical transition. The pipeline of genome-wide dynamic network analysis was performed on the processed data with 14 combinations and a critical transition state of flower development was detected (Fig. 7). The critical transition state detected during the formation of flowers corresponded to the development of specific floral organs (anther and pistil) in rice [61].

Not only that, we also detected the DNB biomarkers with 206 genes that may herald the imminent critical transition during the formation of flowers. The genes detected in the critical transition state were listed in Additional file 10: Figure S3. The anther showed a unique growth characteristic in which most anther-specific genes were expressed only in a particular developmental stage [61]. We found that a pollen-specific gene (LOC4341399) coding for a pollen-specific leucine-rich repeat extensin-like protein was up-regulated in the critical transition. This result indicated that our researches can conduce to reveal the complex regulatory mechanisms of gene expression during anther growth and development as well as pollen germination.

The Gene Ontology (GO) analysis exhibited that protein kinase-encoding genes were significantly enriched among the down-regulated genes (Table 3). The results indicated that several signal transduction pathways involved in protein phosphorylation might undergo complex changes during this critical transition phase. Several

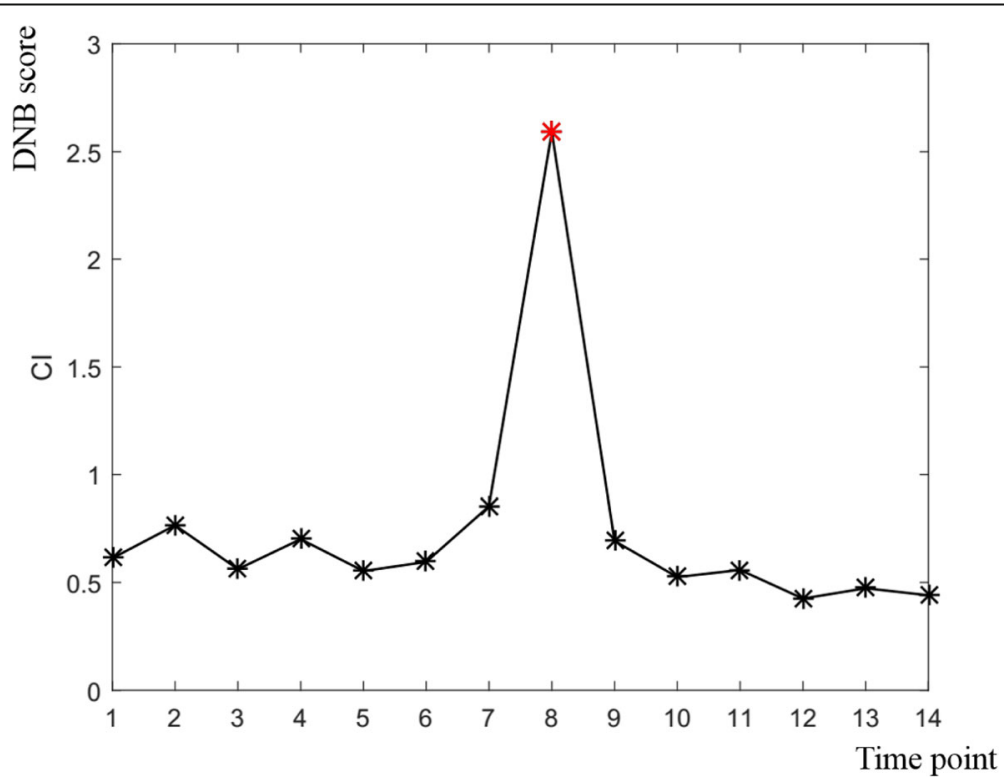

Fig. 7 Identification of the DNB of flower development in rice. The DNB scores at 14 sampling time points is shown in the FIGURE. For the black curve, the DNB score increased sharply from the 7th point and reached the peak at the 8th point. The 8th sampling time point annotated by the red star is designated as a critical transition state, which corresponds to the development of specific floral organs (anther and pistil) in rice 
Table 3 Functional categories for up- and down-regulated genes in the DNB module of rice flower development

\begin{tabular}{|c|c|c|c|}
\hline Gene name & Fold change & Transition state & Description \\
\hline Os04g0417400 & 6.80 & up-regulated & U box domain containing protein. \\
\hline LOC4340069 & 1.93 & up-regulated & DNA-binding protein (Homeodomain-leucine zipper transcription factor) \\
\hline LOC4346839 & 1.82 & up-regulated & Disease resistance protein family protein.disease resistance protein RGA5-like \\
\hline LOC4341399 & 1.74 & up-regulated & pollen-specific leucine-rich repeat extensin-like protein 1 \\
\hline LOC4326659 & 1.59 & up-regulated & Cytochrome P450 monooxygenase CYP72A5 \\
\hline LOC4338660 & 1.56 & up-regulated & UDP-sulfoquinovose synthase, chloroplast precursor (Sulfolipid biosynthesis protein) \\
\hline LOC4345661 & 1.43 & up-regulated & Myb-like protein (transcription factor CSA-like) \\
\hline LOC4324767 & 1.36 & up-regulated & TPR-like domain containing protein. \\
\hline LOC4339280 & 1.36 & up-regulated & Cyclin-like F-box domain containing protein (F-box protein At5g46170) \\
\hline LOC4332901 & 1.32 & up-regulated & Sugar transporter-like protein \\
\hline LOC4324342 & 1.32 & up-regulated & Plant lipid transfer/seed storage/trypsin-alpha amylase inhibitor domain containing protein \\
\hline LOC4351030 & 1.32 & up-regulated & Hypothetical protein \\
\hline LOC4341957 & 1.31 & up-regulated & Macrophage migration inhibitory factor family protein \\
\hline LOC112936214 & 1.32 & up-regulated & eukaryotic cysteine peptidase active site family protein (putative F-box protein At5g15660) \\
\hline LOC4324302 & 1.37 & up-regulated & Myb-related protein 5 (transcription factor MYB59) \\
\hline LOC4338652 & 1.31 & up-regulated & ABC transporter G family member 23 \\
\hline LOC4338416 & 0.79 & down-regulated & ABC-1 domain containing protein (protein ACTIVITY OF BC1 COMPLEX KINASE 3, chloroplastic) \\
\hline LOC4329899 & 0.71 & down-regulated & Galactosyltransferase associated protein kinase p58/GTA (Cell division cycle 2-like 2) \\
\hline LOC4348579 & 0.78 & down-regulated & Protein kinase APK1B (EC 2.7.1.-) \\
\hline LOC4338491 & 0.76 & down-regulated & Ribulose bisphosphate carboxylase \\
\hline LOC4350151 & 0.76 & down-regulated & probable sucrose-phosphate synthase 5 \\
\hline LOC4349274 & 0.75 & down-regulated & Zn-finger, RING domain containing protein (NEP1-interacting protein 1) \\
\hline LOC4335569 & 0.69 & down-regulated & Helicase-like protein \\
\hline LOC4324503 & 0.57 & down-regulated & Ribosomal protein S8 family protein. \\
\hline LOC4335392 & 0.49 & down-regulated & Allergen V5/Tpx-1 related family protein (pathogenesis-related protein 1) \\
\hline LOC9272032 & 0.35 & down-regulated & probable LRR receptor-like serine/ threonine-protein kinase At1g51810 \\
\hline
\end{tabular}

transcription factors including $3 \mathrm{~F}-$ box-, $1 \mathrm{U}$-box-, $2 \mathrm{M}$ YB-family genes were also up-regulated during this critical transition phase. Many studies have indicated that F-box proteins constituted one of the eukaryotic protein families, which played pivotal roles in regulating various developmental processes of plants. For example, a F-box gene DDF1 was a crucial genetic factor with pleiotropic effects in the development of rice floral organs [62]. CSA, a gene encoding the R2R3 MYB transcription factor which preferentially expressed in anther tapetal cells was identified [63]. These researches indicated that the F-box-, U-box-, MYB-family genes detected in this critical transition phase might be play pivotal roles in regulating anther development in rice. Other up- and down-regulated genes also play a crucial role in the process of rice pollen development.

These results supported the conclusions drawn on the development of Arabidopsis flower. Therefore, the transcriptomic profiling analysis using DNB and MARROMI method could provide new insight to characterize the formation of flowers and detect key regulatory factors that might control the transition from initiation to maturation in Arabidopsis flower development.

\section{Discussion}

The formation of flowers is one of the main models for studying the regulation mechanisms of plant growth and development. In past botanical studies, the floral transition was recognized as the progression from vegetative growth to reproductive growth $[34,38]$. Although some network-based bioinformatics analyses attempted to identify the phase transition which indicate the progression from vegetative growth to reproductive, there is no research on identifying the critical transition stage of flower formation from the time of initiation to maturation. Differentiation of floral organs is more complex than other parts of the plant, especially the formation of pollen [61]. In this study, we discovered a critical 
transition stage of Arabidopsis flower development using the DNB theory. It is the first time that DNB model has been used in plant research. We found that the phase transition occurred between the seventh and ninth days of Arabidopsis flower development. The development of flowers on a given inflorescence was uniformly synchronized until day 7 , then the expression of flowering related genes has changed dramatically. Not only that, we found that this critical transition state detected during the formation of flowers corresponded to the development of specific floral organs (anther). We also detected the DNB members composed by 233 genes that may herald the imminent critical transition during the formation of flowers. Moreover, the interactions between these genes also regulate the critical transition process.

To construct the gene regulatory network controlling the flowering transition, we applied NARROMI algorithm which can reduce the noisy, redundant and indirect regulations on the expression data of the transition-specific genes. We further found that 24 genes in this DNB module participate in stress reaction process, as well as in other floral-related pathways such as gametogenesis and embryo development. Our research here suggests that previously unknown regulatory genes identified in the transition region might be through known regulatory mechanisms to promote the formation of flowers. Therefore, a further study of co-expressed genes in the transition region might answer the connection between coexpressed genes and critical transition.

In addition, the highlight of this article is the effective combination of DNB and NARROMI methods. In contrast to the traditional methods or biomarkers based on differential expressions of molecules, DNB method can identify a critical transition state of a complex biological process based on collective fluctuations and correlations of different metabolic molecules at the network level. Thus, even if there are no significant differential expressions in the before-tran sition state and the critical transition state, we can detect significant differential correlations and deviations in these two states by DNB method [35].

In contrast to the traditional methods to construct the gene regulatory network controlling the flowering transition, NARROMI algorithm can reduce the noisy, redundant and indirect regulations on the expression data of the transition-specific genes. Firstly, NARROMI algorithm can calculate the causal intensity between gene pairs by quantifying the non-linear corre lation mutual information (MI), so that we can reduce interference regulators with low correlation. Then, ordinary differential equation-based recursive optimization (RO) is used to gradually reduce the redundant and indirect regulations. Ultimately, we obtained a topology of a non-linear sparse gene regu latory network by network integration which was the most similar gene regulatory network to the real network. NARROMI algorithm could be regard as a further improvement of the DNB model.

We also performed a similar analysis of temporal gene expression profiling dataset of rice flower development to support the conclusions drawn. Ultimately, we detected a critical transition state of flower development in rice. This critical transition state detected during the formation of flowers corresponded to the development of specific floral organs (anther and pistil) in rice. Not only that, we also detected the DNB members composed by 206 genes that may herald the imminent critical transition during the formation of flowers. We found that the genes detected in this critical transition phase might be play pivotal roles in regulating anther development in rice. These results supported the conclusions drawn on the development of Arabidopsis flowering. In addition to the application of DNB and NARROMI algorithm to detect the critical state of Arabidopsis flowering transition, they can be used to detect the critical transition of any biological process.

\section{Conclusions}

We studied the flower developmental phase transition from the time of initiation to maturation in Arabidopsis using dynamic network biomarker model. The critical transition state of flowering development was detected and a cluster of genes as dynamic network biomarkers controlling the phase transition of flower development from the initiation to maturation were identified. In contrast to the traditional methods based on differential gene expression analysis, our analysis can exploit collective fluctuations and correlations of different metabolites at a network level to identify a critical transition state of a complex biological process. We also detected the dynamic network biomarkers composed of several genes that may herald the imminent critical transition during the formation of flowers. To construct the gene regulatory network controlling the flowering transition, we applied NARROMI algorithm on the expression data of the transition-specific genes. The redundancy reduction technique-based network reconstruction method NARROMI algorithm could be regard as a further improvement of the dynamic network biomarker model, which can reduce noisy and indirect regulations to improve the accuracy of the network inference. Our research suggests that the critical transition state detected during the formation of flowers and key regulators identified in the transition might answer the complex mechanisms of floral organ formation. The bioinformatics analysis used in this work can also be used to detect the critical state of any biological process. 


\section{Methods}

\section{Gene expression data collection}

The Arabidopsis flower developmental gene expression profiling dataset was downloaded from the NCBI Gene Expression Omnibus (GEO) database under the accession number GSE64581 (www.ncbi.nlm.nih.gov/geo). The dataset includes 14 different time-points with 3 replicates of gene expression data from initiation to maturity, i.e. 0d, $1 \mathrm{~d}, 1.5 \mathrm{~d}, 2 \mathrm{~d}, 2.5 \mathrm{~d}, 3 \mathrm{~d}, 3.5 \mathrm{~d}, 4 \mathrm{~d}, 4.5 \mathrm{~d}, 5 \mathrm{~d}, 7 \mathrm{~d}, 9 \mathrm{~d}, 11 \mathrm{~d}$ and 13d (Fig. 6a). The data was downloaded from GEO database already pre-processed, and we followed procedure outlined in Ryan et al., 2015 and some other protocol. Ryan et al.'s article is an Open Access article distributed under the terms of the Creative Commons Attribution License (http://creativecommons.org/licenses/by/4.0).

The data was downloaded from GEO database was a time-courses gene expression matrix. The horizontal row indicated the expression value of one gene in different samples, and the column represented the expression value of the gene pool of one sample. We used the function 'genelowvalfilter' in MATLAB to filter genes. After removing those genes whose expression values were less than a certain threshold, we used the remaining genes for further processing. Because some of the transcriptional changes are caused by specific gene regulatory events or tremendous alterations in floral size and morp hology during the flower development, we compared the gene expression level between consecutive as well as neighbor (within a $2 \mathrm{~d}$ time interval) time-points to minimize the effect of morphological changes [8].

\section{Network inference}

In general, a mathematical model based on mass action kinetics and Michaelis-Menten kinetics can describe the transcriptional regulation process [64]. However, the noise inherited in the gene expression data can decrease the performance of these models [65]. Therefore, to improve the accuracy of the network inference, NARROMI algorithm was used to reduce noisy, redundant and indirect regulations [29]. It initially calculates the causal strength between gene pairs by quantifying the non-linear correlation mutual information (MI), which can reduce noisy regulations in gene expression data [66]. Then, ordinary differential equation-based recursive optimization ( $\mathrm{RO}$ ) is used to gradually reduce the redundant and indirect regulations to obtain a final topology of a non-linear sparse gene regulatory network (Fig. 6c). The script that was used to apply the NARROMI algorithm was listed in Additional file 11: Script 1. By comparing with GENIE3, ARACNE etc., NARROMI outperformed these popular methods in most cases, thereby verifying its effectiveness [29].

To obtain a more reliable gene co-expression network, a composite index is proposed combining mutual
information(MI) and recursive optimization(RO)-based estimation of parameters:

$$
\beta=\operatorname{sign}\left(\beta^{\mathrm{RO}}\right)\left(\omega\left|\beta^{\mathrm{RO}}\right|+(1-\omega) \beta^{\mathrm{MI}}\right)
$$

where $\beta^{\mathrm{MI}}$ is the MI value which is positive, $\beta^{\mathrm{RO}}$ is the regulatory strength (positive or negative) inferred by RO algorithm, $\operatorname{sign}\left(\beta^{\mathrm{RO}}\right)$ is the sign $( \pm)$ of $\beta^{\mathrm{RO}},\left|\beta^{\mathrm{RO}}\right|$ is the absolute of $\beta^{\mathrm{RO}}$ and parameter $\omega$ is the weighting coefficient. The final regulatory strength is decided by the parameter $\beta$, and the network topology is then determined.

\section{GO enrichment and visualization}

The identified DNB members were analyzed for functional enrichment analysis by $\mathrm{R}$ package topGO 2.30.1. The topGO package provided tools for testing GO terms while accounting for the topology of the GO graph. Different test statistics and different methods for eliminating local similarities and dependencies between GO terms can be implemented and applied. The enrichment analysis process consists of input of normalized gene expression measurements, gene-wise correlation or differential expression analysis, enrichment analysis of GO terms, interpretation and visualization of the results. The data preparation process is critical before running the enrichment test. The user needs to provide the gene universe, GO annotations and either a criteria for selecting interesting genes (e.g. differentially expressed genes) from the gene universe or a score associated with each gene. The GO database used in our research was a set of annotation maps describing the entire Gene Ontology assembled using data from GO Version: 3.5.0.

Moreover, we also analyzed key regulatory factors and key metabolic pathways that were closely related to DNB of Arabidopsis flower development from initiation to maturation time (Fig. 6d). The results of the gene regulatory networks were imported in Cytoscape (www.cytoscape.org) for visualization.

\section{Regulatory circuits and regulators prediction}

We further predicted the regulatory circuits and regulators controlling the critical transition based on the Arabidopsis Gene Regulatory Information Server (AGRIS; http://arabidopsis.med.ohio-state.edu) [67]. We can obtain a comprehensive resource for Arabidopsis gene regulatory studies from the AGRIS. There are three interlinked databases, AtTFDB, AtcisDB and AtRegNet. The updated and comprehensive information on transcription factors (TFs) could be obtain from AtTFDB, which is the key to study the Arabidopsis gene regulatory networks. 


\section{Additional files}

Additional file 1: Figure S4. Comparison results of the DNB-based method, bootstrap analysis. (DOCX $313 \mathrm{~kb}$ )

Additional file 2: Figure S1. List of genes for the DNB module during Arabidopsis flower development. (TXT $2 \mathrm{~kb}$ )

Additional file 3: Figure S2. Detailed description of the identified DNB module for Arabidopsis flower development. (TXT $95 \mathrm{~kb}$ )

Additional file 4: Table S1. Functional categories for the DNB members of Arabidopsis flower development. (TXT $7 \mathrm{~kb}$ )

Additional file 5: Table S3. Network inference file. (TXT $32 \mathrm{~kb}$ )

Additional file 6: Table S4. The degree of correlation between each pair of DNB genes during Arabidopsis flower development. (TXT 7 kb)

Additional file 7: Table S5. Node properties file for the network. (TXT $4 \mathrm{~kb}$ )

Additional file 8: Table S6. The gene regulatory network Cytoscape file. (TXT $7 \mathrm{~kb}$ )

Additional file 9: Table S2. The DNB members of Arabidopsis flower development enriched GO terms and metabolic pathways. (TXT 2 kb)

Additional file 10: Figure S3. The DNB members of flower

development in rice. (TXT $9 \mathrm{~kb}$ )

Additional file 11: Script 1. Tair_DNB_narromi. (DOCX 14 kb)

\section{Abbreviations}

ABA: Abscisic acid; CBC: Cap-binding protein complex; DNB: Dynamic network biomarker; ECA1: Early Culture Abundant 1; GRN: Gene regulatory network; LEA: Late embryogenesis Abundant; MI: Mutual information; PCC: Pearson correlation coefficient; RO: Recursive optimization

\section{Acknowledgements}

We thank Dr. P.T. Ryan (Smurfit Institute of Genetics, Trinity College Dublin) for providing Arabidopsis floral gene expression data and members of our labs for discussion. We also thank O.O. Collins (University of Chinese Academy of Sciences) for providing invaluable assistance in writing this article.

\section{Funding}

This work was supported by grants from National Natural Sciences Foundation of China(NSFC), no.61402457. CAS Pioneer Hundred Talents Program and the National Project of Cause Control Theory, no.1716315XJ00200303.

\section{Availability of data and materials}

The data sets are included within the article and its Additional files.

\section{Authors' contributions}

X.Z. designed the research; F.Z. performed the research, F.Z. analyzed the data; L.C., Z.J., X.L. and A.Z. provided many critical suggestions and approved the manuscript; F.Z. and X.Z. wrote the article. All authors read and approved the final manuscript.

\section{Ethics approval and consent to participate}

Not applicable.

\section{Consent for publication}

Not applicable.

\section{Competing interests}

The authors declare that they have no competing interests.

\section{Publisher's Note}

Springer Nature remains neutral with regard to jurisdictional claims in published maps and institutional affiliations.

\section{Author details}

Key Laboratory of Plant Germplasm Enhancement and Specially Agriculture, Wuhan Botanical Garden, Chinese Academy of Sciences, Wuhan 430074, China. ${ }^{2}$ University of Chinese Academy of Sciences, Beijing 10049, China. ${ }^{3}$ Key Laboratory of Systems Biology, Innovation Center for Cell Signaling Network, Institute of Biochemistry and Cell Biology, Shanghai Institutes for Biological Sciences, Chinese Academy of Sciences, Shanghai 200031, China.

Received: 20 July 2018 Accepted: 4 December 2018

Published online: 07 January 2019

\section{References}

1. Rougvie AE. Intrinsic and extrinsic regulators of developmental timing: from miRNAs to nutritional cues. Development. 2005;132(17):3787-98.

2. Amasino R. Seasonal and developmental timing of flowering. Plant J. 2010; 61(6):1001-13

3. Kubota A, Kita S, Ishizaki K, Nishihama R, Yamato KT, Kohchi T. Co-option of a photoperiodic growth-phase transition system during land plant evolution. Nat Commun. 2014;5:3668.

4. Jia H, Suzuki M, McCarty DR. Regulation of the seed to seedling developmental phase transition by the LAFL and VAL transcription factor networks. Wiley Interdiscipl Rev Dev Biol. 2014;3(1):135-45.

5. Moose SP, Sisco PH. Glossy15 controls the epidermal juvenile-to-adult phase transition in maize. Plant Cell. 1994:6(10):1343-55.

6. Araki T. Transition from vegetative to reproductive phase. Curr Opin Plant Biol. 2001:4(1):63-8.

7. Chen M, Thelen JJ. The plastid isoform of triose phosphate isomerase is required for the postgerminative transition from heterotrophic to autotrophic growth in Arabidopsis. Plant Cell. 2010;22(1):77-90.

8. Ryan PT, O'Maoileidigh DS, Drost HG, Kwasniewska K, Gabel A, Grosse I, Graciet E, Quint M, Wellmer F. Patterns of gene expression during Arabidopsis flower development from the time of initiation to maturation. BMC Genomics. 2015;16:488.

9. Huijser P, Schmid M. The control of developmental phase transitions in plants. Development. 2011;138(19):4117-29.

10. Soltis DE, Ma H, Frohlich MW, Soltis PS, Albert VA, Oppenheimer DG, Altman NS, dePamphilis C, Leebens-Mack J. The floral genome: an evolutionary history of gene duplication and shifting patterns of gene expression. Trends Plant Sci. 2007;12(8):358-67.

11. Wellmer F, Riechmann JL. Gene networks controlling the initiation of flower development. Trends Genet. 2010;26(12):519-27.

12. Gong $X$, Shen $L$, Peng $Y Z$, Gan $Y, Y u ~ H$. DNA topoisomerase lalpha affects the floral transition. Plant Physiol. 2017;173(1):642-54

13. Wang L, Kong D, Lv Q, Niu G, Han T, Zhao X, Meng S, Cheng Q, Guo S, Du J, et al. Tetrahydrofolate modulates floral transition through epigenetic silencing. Plant Physiol. 2017:174(2):1274-84

14. Jiang L, Li D, Jin L, Ruan Y, Shen WH, Liu C. Histone lysine methyltransferases BnaSDG8.A and BnaSDG8.C are involved in the floral transition in Brassica napus. Plant J. 2018;95(4):672-85.

15. Brambilla V, Martignago D, Goretti D, Cerise M, Somssich M, de Rosa M, Galbiati F, Shrestha R, Lazzaro F, Simon R, et al. Antagonistic transcription factor complexes modulate the floral transition in Rice. Plant Cell. 2017:29(11):2801-16.

16. Bellaire A, Ischebeck T, Staedler Y, Weinhaeuser I, Mair A, Parameswaran S, Ito T, Schonenberger J, Weckwerth W. Metabolism and development integration of micro computed tomography data and metabolite profiling reveals metabolic reprogramming from floral initiation to silique development. New Phytol. 2014;202(1):322-35.

17. Putterill J, Laurie R, Macknight R. It's time to flower: the genetic control of flowering time. Bioessays. 2004;26(4):363-73.

18. Huang J, Vendramin S, Shi L, McGinnis KM. Construction and optimization of a large gene Coexpression network in maize using RNA-Seq data. Plant Physiol. 2017;175(1):568-83.

19. Silva AT, Ribone PA, Chan RL, Ligterink W, Hilhorst HW, Predictive Coexpression A. Network identifies novel genes controlling the seed-toseedling phase transition in Arabidopsis thaliana. Plant Physiol. 2016; 170(4):2218-31

20. Vesty EF, Saidi Y, Moody LA, Holloway D, Whitbread A, Needs S, Choudhary A, Burns B, McLeod D, Bradshaw SJ, et al. The decision to germinate is regulated by divergent molecular networks in spores and seeds. New Phytol. 2016;211(3):952-66. 
21. Chen P, Liu R, Li Y, Chen L. Detecting critical state before phase transition of complex biological systems by hidden Markov model. Bioinformatics. 2016; 32(14):2143-50.

22. Harkey AF, Watkins JM, Olex AL, DiNapoli KT, Lewis DR, Fetrow JS, Binder BM, Muday GK. Identification of transcriptional and receptor networks that control root responses to ethylene. Plant Physiol. 2018;176(3):2095-118.

23. Hong $S$, Chen $X$, Jin L, Xiong M. Canonical correlation analysis for RNA-seq co-expression networks. Nucleic Acids Res. 2013;41(8):e95.

24. Wang J, Chen B, Wang Y, Wang N, Garbey M, Tran-son-Tay R, Berceli SA, Wu $R$. Reconstructing regulatory networks from the dynamic plasticity of gene expression by mutual information. Nucleic Acids Res. 2013;41(8):e97.

25. Basso K, Margolin AA, Stolovitzky G, Klein U, Dalla-Favera R, Califano A. Reverse engineering of regulatory networks in human B cells. Nat Genet. 2005;37(4):382-90.

26. Gardner TS, di Bernardo D, Lorenz D, Collins JJ. Inferring genetic networks and identifying compound mode of action via expression profiling. Science. 2003:301(5629):102-5.

27. Cantone I, Marucci L, lorio F, Ricci MA, Belcastro V, Bansal M, Santini S, di Bernardo M, di Bernardo D, Cosma MP. A yeast synthetic network for in vivo assessment of reverse-engineering and modeling approaches. Cell. 2009; 137(1):172-81.

28. Honkela A, Girardot C, Gustafson EH, Liu YH, Furlong EE, Lawrence ND, Rattray M. Model-based method for transcription factor target identification with limited data. Proc Natl Acad Sci U S A. 2010;107(17): 7793-8.

29. Zhang X, Liu K, Liu ZP, Duval B, Richer JM, Zhao XM, Hao JK, Chen L. NARROMI: a noise and redundancy reduction technique improves accuracy of gene regulatory network inference. Bioinformatics. 2013;29(1):106-13.

30. Zhang X, Zhao XM, He K, Lu L, Cao Y, Liu J, Hao JK, Liu ZP, Chen L. Inferring gene regulatory networks from gene expression data by path consistency algorithm based on conditional mutual information. Bioinformatics. 2012; 28(1):98-104.

31. Zhang X, Zhao J, Hao JK, Zhao XM, Chen L. Conditional mutual inclusive information enables accurate quantification of associations in gene regulatory networks. Nucleic Acids Res. 2015;43(5):e31.

32. Zhao J, Zhou Y, Zhang X, Chen L. Part mutual information for quantifying direct associations in networks. Proc Natl Acad Sci U S A. 2016;113(18):5130-5.

33. Bassel GW, Lan H, Glaab E, Gibbs DJ, Gerjets T, Krasnogor N, Bonner AJ, Holdsworth MJ, Provart NJ. Genome-wide network model capturing seed germination reveals coordinated regulation of plant cellular phase transitions. Proc Natl Acad Sci U S A. 2011;108(23):9709-14.

34. He F, Zhou Y, Zhang Z. Deciphering the Arabidopsis floral transition process by integrating a protein-protein interaction network and gene expression data. Plant Physiol. 2010;153(4):1492-505

35. Dinh JL, Farcot E, Hodgman C. The logic of the floral transition: reverseengineering the switch controlling the identity of lateral organs. PLoS Comput Biol. 2017:13(9):e1005744.

36. Bouyer D, Roudier F, Heese M, Andersen ED, Gey D, Nowack MK, Goodrich J, Renou JP, Grini PE, Colot V, et al. Polycomb repressive complex 2 controls the embryo-to-seedling phase transition. PLoS Genet. 2011;7(3):e1002014.

37. Wils CR, Kaufmann K. Gene-regulatory networks controlling inflorescence and flower development in Arabidopsis thaliana. Biochim Biophys Acta. 2017;1860(1):95-105

38. Dong Z, Danilevskaya O, Abadie T, Messina C, Coles N, Cooper M. A gene regulatory network model for floral transition of the shoot apex in maize and its dynamic modeling. PLoS One. 2012;7(8):e43450.

39. Varala K, Marshall-Colon A, Cirrone J, Brooks MD, Pasquino AV, Leran S, Mittal S, Rock TM, Edwards MB, Kim GJ, et al. Temporal transcriptional logic of dynamic regulatory networks underlying nitrogen signaling and use in plants. Proc Natl Acad Sci U S A. 2018;115(25):6494-9.

40. Greenham K, McClung CR. Time to build on good design: resolving the temporal dynamics of gene regulatory networks. Proc Natl Acad Sci U S A. 2018;115(25):6325-7.

41. Chen L, Liu R, Liu ZP, Li M, Aihara K. Detecting early-warning signals for sudden deterioration of complex diseases by dynamical network biomarkers. Sci Rep. 2012;2:342

42. Liu R, Wang X, Aihara K, Chen L. Early diagnosis of complex diseases by molecular biomarkers, network biomarkers, and dynamical network biomarkers. Med Res Rev. 2014;34(3):455-78.

43. Ge J, Fan XY, Xue XL, Li GP, Zhong SS, Shen X, Yin HY, Chen LN. Discovery of dynamical network biomarkers (DNB) during the progression of atherosclerosis using multiple omic techniques and systems biology. J Am Coll Cardiol. 2017;70(16):C34-5.

44. Sa R, Zhang W, Ge J, Wei X, Zhou Y, Landzberg DR, Wang Z, Han X, Chen L, Yin $\mathrm{H}$. Discovering a critical transition state from nonalcoholic hepatosteatosis to nonalcoholic steatohepatitis by lipidomics and dynamical network biomarkers. J Mol Cell Biol. 2016;8(3):195-206.

45. Li MY, Zeng T, Liu R, Chen LN. Detecting tissue-specific early warning signals for complex diseases based on dynamical network biomarkers: study of type 2 diabetes by cross-tissue analysis. Brief Bioinform. 2014;15(2):229-43.

46. Chen P, Liu R, Chen L, Aihara K. Identifying critical differentiation state of MCF-7 cells for breast cancer by dynamical network biomarkers. Front Genet. 2015;6:252.

47. Smyth DR, Bowman JL, Meyerowitz EM. Early flower development in arabidopsis. Plant Cell. 1990;2(8):755-67.

48. Anders S, Huber W. Differential expression analysis for sequence count data. Genome Biol. 2010;11(10):12.

49. Sakuraba Y, Kim YS, Han SH, Lee BD, Paek NC. The Arabidopsis transcription factor NAC016 promotes drought stress responses by repressing AREB1 transcription through a trifurcate feed-forward regulatory loop involving NAP. Plant Cell. 2015;27(6):1771-87.

50. Kierzkowski D, Kmieciak M, Piontek P, Wojtaszek P, SzweykowskaKulinska Z, Jarmolowski A. The Arabidopsis CBP20 targets the capbinding complex to the nucleus, and is stabilized by CBP80. Plant J. 2009;59(5):814-25

51. Kong X, Ma L, Yang L, Chen Q, Xiang N, Yang Y, Hu X. Quantitative proteomics analysis reveals that the nuclear cap-binding complex proteins arabidopsis CBP20 and CBP80 modulate the salt stress response. J Proteome Res. 2014;13(5):2495-510.

52. Jager K, Fabian A, Tompa G, Deak C, Hohn M, Olmedilla A, Barnabas B, Papp I. New phenotypes of the drought-tolerant cbp20 Arabidopsis thaliana mutant have changed epidermal morphology. Plant Biol (Stuttg). 2011;13(1):78-84.

53. Krishnaswamy S, Verma S, Rahman MH, Kav NN. Functional characterization of four APETALA2-family genes (RAP2.6, RAP2.6L, DREB19 and DREB26) in Arabidopsis. Plant Mol Biol. 2011;75(1-2):107-27.

54. Zhu Q, Zhang J, Gao X, Tong J, Xiao L, Li W, Zhang H. The Arabidopsis AP2/ ERF transcription factor RAP2.6 participates in ABA, salt and osmotic stress responses. Gene. 2010:457(1-2):1-12.

55. Nag A, Yang YZ, Jack T. DORNROSCHEN-LIKE, an AP2 gene, is necessary for stamen emergence in Arabidopsis. Plant Mol Biol. 2007;65(3):219-32.

56. Yang Y, Liang T, Zhang L, Shao K, Gu X, Shang R, Shi N, Li X, Zhang P, Liu H. UVR8 interacts with WRKY36 to regulate HY5 transcription and hypocotyl elongation in Arabidopsis. Nat Plants. 2018;4(2):98-107.

57. O'Maoileidigh DS, Graciet E, Wellmer F. Gene networks controlling Arabidopsis thaliana flower development. New Phytol. 2014;201(1):16-30.

58. Chen F, Zhang X, Liu X, Zhang L. Evolutionary analysis of MIKCc-type MADSbox genes in gymnosperms and angiosperms. Front Plant Sci. 2017:8:895.

59. Zhang DS, Liang WQ, Yuan Z, Li N, Shi J, Wang J, Liu YM, Yu WJ, Zhang DB. Tapetum degeneration retardation is critical for aliphatic metabolism and gene regulation during rice pollen development. Mol Plant. 2008; 1(4):599-610.

60. Chandler JW. The hormonal regulation of flower development. J Plant Growth Regul. 2010;30(2):242-54

61. Sato $Y$, Antonio B, Namiki N, Motoyama R, Sugimoto K, Takehisa H, Minami $\mathrm{H}$, Kamatsuki K, Kusaba M, Hirochika H, et al. Field transcriptome revealed critical developmental and physiological transitions involved in the expression of growth potential in japonicarice. BMC Plant Biol. 2011;11(1):10.

62. Duan Y, Li S, Chen Z, Zheng L, Diao Z, Zhou Y, Lan T, Guan H, Pan R, Xue Y, et al. Dwarf and deformed flower 1, encoding an F-box protein, is critical for vegetative and floral development in rice (Oryza sativa L.). Plant J. 2012; 72(5):829-42.

63. Zhang H, Liang W, Yang X, Luo X, Jiang N, Ma H, Zhang D. Carbon starved anther encodes a MYB domain protein that regulates sugar partitioning required for rice pollen development. Plant Cell. 2010;22(3):672-89.

64. Tian TH, Burrage K, Burrage PM, Carletti M. Stochastic delay differential equations for genetic regulatory networks. J Comput Appl Math. 2007; 205(2):696-707.

65. Lozoya OA, Santos JH, Woychik RP. A Leveraged Signal-to-Noise ratio (LSTNR) method to extract differentially expressed genes and multivariate patterns of expression from Noisy and low-replication RNAseq data. Front Genet. 2018;9:24. 
66. Steuer R, Kurths J, Daub CO, Weise J, Selbig J. The mutual information: detecting and evaluating dependencies between variables. Bioinformatics. 2002;18:5231-40

67. Davuluri RV, Sun H, Palaniswamy SK, Matthews N, Molina C, Kurtz M, Grotewold E. AGRIS: Arabidopsis gene regulatory information server, an information resource of Arabidopsis cis-regulatory elements and transcription factors. BMC Bioinf. 2003;4:11.

Ready to submit your research? Choose BMC and benefit from:

- fast, convenient online submission

- thorough peer review by experienced researchers in your field

- rapid publication on acceptance

- support for research data, including large and complex data types

- gold Open Access which fosters wider collaboration and increased citations

- maximum visibility for your research: over $100 \mathrm{M}$ website views per year

At $B M C$, research is always in progress.

Learn more biomedcentral.com/submissions 